\title{
Féeries
}

Études sur le conte merveilleux, XVII ${ }^{\mathrm{e}} \mathrm{XIX} \mathrm{X}^{\mathrm{e}}$ siècle

Le conte, la scène

\section{The Arabian Nights Reader}

Edited by Ulrich Marzolph, Wayne State University Press, Detroit, 2006, 364 pages et un index.

Jean-François Perrin

\section{(2) OpenEdition}

1 Journals

Édition électronique

URL : http://journals.openedition.org/feeries/493

DOI : $10.4000 /$ feeries.493

ISSN : 1957-7753

Éditeur

UGA Éditions/Université Grenoble Alpes

Édition imprimée

Date de publication : 1 octobre 2007

Pagination : 260-262

ISBN : 978-2-84310-101-4

ISSN : 1766-2842

\section{Référence électronique}

Jean-François Perrin, «The Arabian Nights Reader », Féeries [En ligne], 4 | 2007, mis en ligne le 18 décembre 2013, consulté le 24 septembre 2020. URL : http://journals.openedition.org/feeries/493 ; DOI : https://doi.org/10.4000/feeries.493

Ce document a été généré automatiquement le 24 septembre 2020.

(c) Féeries 


\section{The Arabian Nights Reader}

Edited by Ulrich Marzolph, Wayne State University Press, Detroit, 2006, 364 pages et un index.

Jean-François Perrin

1 Cet ouvrage procuré par Ulrich Marzolph, professeur d'études islamiques à l'Université de Göttingen, s'inscrit dans un vaste programme international de mise à disposition des recherches modernes sur le conte merveilleux et sur les Mille et Une Nuits, dont l'envergure n'est comparable qu'aux entreprises anciennes de Victor Chauvin et de René Basset ${ }^{1}$. C'est ainsi qu'après avoir récemment publié, en collaboration avec Richard van Leeuwen, une somme sans équivalent sur les Nuits et leur tradition littéraire : The Arabian Nights Encyclopedia², ce spécialiste renommé présente maintenant une anthologie de seize études marquantes, voire désormais "classiques», sur le fameux recueil, parues (ou traduites) en langue anglaise au cours du dernier demi-siècle. Entreprise justifiée, selon la substantielle introduction qu'il y joint, par le fait que c'est précisément durant cette période que nombre de questions scientifiques et critiques ouvertes depuis le XIX siècle ont été renouvelées ou ont trouvé réponse. Les domaines de recherche abordés concernent principalement l'histoire du recueil et les problèmes de poétique; s'y joignent une série de monographies et une étude sur l'influence des Nuits dans la littérature arabe contemporaine.

2 Six études sont donc consacrées aux aspects historiques. Celle de Muhsin Jassim Ali : "The Growth of Scholarly Interest in the Arabian Nights ( (1980), concerne la réception critique des Nuits depuis le $\mathrm{xIX}^{\mathrm{e}}$ siècle (Lane, Payne, Clouston) jusqu'aux travaux plus récents de Mia Gerhardt, Tzvetan Todorov et John Barth. L'article de Nabia Abbott : «A Ninth-Century Fragment of the Thousand Nights: New Light on the Early History of the Arabian Nights» (1949), était incontournable dans une telle anthologie, puisqu'il porte sur la découverte en 1947 d'un fragment d'une version des Nuits remontant au IX ${ }^{\mathrm{e}}$ siècle, attestant ainsi l'existence du recueil dès cette époque. Dans une perpective proche, le travail de Solomon D. Goiten : «The Oldest Documentary Evidence for the Title Alf Laila wa-Laila» (1958), démontre que ce titre est déjà celui du recueil vers le milieu du XII ${ }^{\mathrm{e}}$ siècle, soit avant l'époque du plus ancien manuscrit connu, puisqu'il apparaît dans un carnet ayant appartenu à un savant du Caire qui vendait et louait des livres. Heinz 
Grotzfeld quant à lui, dans un article intitulé : «Neglected Conclusions of the Arabian Nights: Gleanings in Forgotten an Overlooked Recensions » (1985), reprend l'histoire des versions négligées de la conclusion du recueil, montrant que l'autorité accordée à la version égyptienne dite recension Zotemberg (la conclusion y montre la conteuse implorant le pardon du roi en arguant des enfants qu'elle lui a donnés), a fait oublier l'existence dans certaines versions manuscrites bien antérieures, de conclusions où c'est la réflexion du roi sur les récits de Scheherazade qui l'amène à se remettre en question. Dans une autre étude: "The Age of the Galland Manuscript of the Nights: Numismatic Evidence for Dating a Manuscript? »(1996-1997), le même savant repousse de près d'un demi-siècle la datation du manuscrit Galland, à partir de considérations numismatiques sur le nom d'une pièce de monnaie mentionnée par l'Histoire du savant juif dans le cycle des Bossus : ainsi, le plus ancien manuscrit connu des Nuits ne serait pas antérieur à la seconde moitié du $\mathrm{xv}^{\mathrm{e}}$ siècle, contrairement à la thèse de Mahdi son éditeur moderne, qui le situe au XIV siècle. On retrouve d'ailleurs dans cette anthologie, sous la plume du même Muhsin Mahdi, une discussion célèbre: "The Sources of Galland's Nuits » (1993), sur les mystères et les ombres de la traduction Galland et sur sa fidélité philologique à son (ses ?) manuscrit(s) : selon lui, Galland a certes puissamment contribué à l'évolution de la prose narrative en Occident, mais il a corrompu sa source.

$\mathrm{Au}$ chapitre des problèmes de poétique du genre, c'est l'influence sur les Nuits de la littérature grecque - et tout particulièrement le roman -, qui fait l'objet de l'étude de Gustave E. von Grunebaum : "Greek Form Elements in the Arabian Nights » (1942); et c'est encore la dimension romanesque qui retient l'attention de Peter Heath: "Romance as Genre in The Thousands an One Nights» (1987-1988). On ne présente pas au lecteur français la fameuse étude de Tzvetan Todorov: «Les Hommes-récits » (1971), montrant que le contrat : "un récit contre une vie » ne régit pas seulement le cadre mais aussi l'ensemble du recueil, ainsi envisagé comme une prodigieuse machine narrative. Au chapitre des monographies, on lira de Roger Allen une analyse de la technique de l'encadrement et du commentaire dans le recueil, saisie à travers le conte des Trois pommes: "An Analysis of the "Tale of the Three Apples" from The Thousand and One Nights» (1984). De Jamel Eddine Bencheikh, est donnée la version anglaise d'une de ces étude à la fois savantes et poétiques dont il avait le secret, portant ici sur l'ordre de vérité propre à l'imaginaire et à la fiction dans les Nuits: "Historical an Mythical Baghdad in the Tale of 'Ali b. Bakkar and Shams al-Nahar, or the Resurgence of the Imaginary » (1997). Quant à l'histoire de la Cité de Cuivre, elle est l'allégorie du monde comme (triste) mirage selon l'étude d'Andras Hamori : « An Allegory from the Arabian Nights: The City of Brass » (1974). Une seconde étude de Muhsin Mahdi étudie la réécriture créative d'un récit historique $\mathrm{du} \mathrm{x}^{\mathrm{e}}$ siècle par les compilateurs des Nuits: «From History to Fiction: The Tale Told by the King's Steward» (1984). Et c'est aux voyages de Sindbad lus comme une parabole cynique à l'usage du Pouvoir, que s'attache Peter D. Molan: "Sindbad the Sailor: A Commentary on the Ethics of Violence" (1984). À un étiage plus contemporain, c'est à l'histoire critique de l'interprétation sociale et politique du conte d'Aladdin à l'écran par le dessin animé (Disney, 1992) et le cinéma (Douglas Fairbanks - 1924, et Alexander Korda - 1940), que s'attache Michael Cooperson: "The Monstrous Births of 'Aladdin'» (1994). L'ouvrage se clôt par une étude récente de Fedwa Malti-Douglas: «Sharazad Feminist» (1997), consacrée aux transformations complexes de la figure de la conteuse dans la littérature contemporaine, à travers deux œuvres féministes: Scheherazade Retold de l'américaine Ethel Johnston Phelps, et The Fall of the Imam de l'Égyptienne Nawal El Saadawi. 
4 L'appareil de notes de chaque étude est restitué et un index des noms et des titres clôt le volume.

5 Complétant utilement la précieuse synthèse de R. Irwin ${ }^{3}$, cette anthologie procure un accès direct à quelques-uns des moments les plus déterminants de la recherche scientifique et littéraire moderne sur les Mille et Une Nuits; on peut certes regretter que le principe de recueillir uniquement des études déjà publiées en langue anglaise empêche la mise à disposition d'autres ressources non traduites - et pour le domaine français par exemple, de certains travaux pionniers d'André Miquel ou de Claude Bremond; cependant, par son caractère interdisciplinaire et la variété des approches présentées, l'ouvrage nourrira efficacement les études à venir des contes merveilleux de Scheherazade.

\section{NOTES}

1. Voir dans Féeries $\mathrm{n}^{\circ}$ 3, mon compte rendu des deux volumes de ses Mille et un contes, récits et légendes arabes, réédités aux éditions Corti (2005).

2. The Arabian Nights Encyclopedia, Santa Barbara, CA: ABC-CLIO (2004).

3. The Arabian Nights, a Companion, rééd. Tauris (2004), voir le compte-rendu de J-P. Sermain dans Féeries $\mathrm{n}^{\circ} 2$. 